\title{
Levels of Circulating Microparticles in Lung Cancer Patients and Possible Prognostic Value
}

\author{
Chia-Cheng Tseng, ${ }^{1}$ Chin-Chou Wang, ${ }^{1,2}$ Huang-Chih Chang, ${ }^{1}$ Tzu-Hsien Tsai, ${ }^{3}$ \\ Li-Teh Chang, ${ }^{4}$ Kuo-Tung Huang, ${ }^{1}$ Steve Leu, ${ }^{5}$ Chia-Hung Yen, ${ }^{6}$ Shih-Feng Liu, ${ }^{1}$ \\ Chih-Hung Chen, ${ }^{7}$ Cheng-Ta Yang, ${ }^{8}$ Hon-Kan Yip, ${ }^{3,5}$ and Meng-Chih Lin ${ }^{1}$ \\ ${ }^{1}$ Division of Pulmonary and Critical Care Medicine, Department of Internal Medicine, Kaohsiung Chang Gung Memorial Hospital, \\ Chang Gung University College of Medicine, Kaohsiung 83301, Taiwan \\ ${ }^{2}$ Department of Public Health, Kaohsiung Medical University, Kaohsiung 83301, Taiwan \\ ${ }^{3}$ Division of Cardiology, Department of Internal Medicine, Kaohsiung Chang Gung Memorial Hospital, \\ Chang Gung University College of Medicine, No. 123 Ta Pei Road, Niao Sung District, Kaohsiung 83301, Taiwan \\ ${ }^{4}$ Basic Science, Nursing Department, Meiho University, Pingtung 900, Taiwan \\ ${ }^{5}$ Center for Translational Research in Biomedical Sciences, Kaohsiung Chang Gung Memorial Hospital, \\ Chang Gung University College of Medicine, Kaohsiung 83301, Taiwan \\ ${ }^{6}$ Department of Life Science, National Pingtung University of Science and Technology, Pingtung 900, Taiwan \\ ${ }^{7}$ Divisions of General Medicine, Kaohsiung Chang Gung Memorial Hospital and Chang Gung University College of Medicine, \\ No. 123 Ta Pei Road, Niao Sung District, Kaohsiung 83301, Taiwan \\ ${ }^{8}$ Department of Pulmonary and Critical Care Medicine, Chang Gung Memorial Hospital, Chang Gung University College of Medicine, \\ Taoyuan 333, Taiwan
}

Correspondence should be addressed to Hon-Kan Yip; yiphonkan@hotmail.com.tw

Received 27 February 2013; Accepted 26 August 2013

Academic Editor: Mariann Harangi

Copyright (C) 2013 Chia-Cheng Tseng et al. This is an open access article distributed under the Creative Commons Attribution License, which permits unrestricted use, distribution, and reproduction in any medium, provided the original work is properly cited.

Background. Endothelial-derived microparticles (EDMPs) and platelet-derived microparticles (PDMPs) have been reported to be increasing in various diseases including malignant diseases. Here, we investigated whether these MPs may be useful biomarkers for predicting lung cancer (LC) disease status, cell type, or metastasis. Methods and Results. One hundred and thirty LC patients were prospectively enrolled into the study between April 2011 and February 2012. Flow cytometric analysis demonstrated that the circulating levels of platelet-derived activated MPs (PDAc-MPs), platelet-derived apoptotic MPs (PDAp-MPs), endothelial-derived activated MPs (EDAc-MPs), and endothelial-derived apoptotic MPs (EDAp-MPs) were significantly higher in LC patients than in 30 age- and gender-matched normal control subjects (all $P<0.05$ ). Additionally, circulating level of PDAc-MPs was significantly lower $(P=0.031)$, whereas the circulating levels of the other three biomarkers did not differ (all $P>0.1)$ in early stage versus late stage LC patients. Furthermore, the circulating levels of the four types of MPs did not differ among patients with different disease statuses (i.e., disease controlled, disease progression, and disease without treatment, i.e., fresh case) (all $P>0.2)$ or between patients with or without LC metastasis (all $P>0.5$ ). Moreover, only the circulating level of EDAp-MPs was significantly associated with the different cell types (i.e., squamous cell carcinoma, adenocarcinoma, and small cell carcinoma) of LC $(P=0.045)$. Conclusion. Circulating MP levels are significantly increased in LC patients as compared with normal subjects. Among the MPs, only an increased level of EDAp-MPs was significantly associated with different LC cell types.

\section{Introduction}

Lung cancer (LC) accounts for more cancer deaths than any other tumor type worldwide $[1,2]$. Despite concerted efforts to improve early diagnosis and treatment of LC patients, overall survival rate is still poor [3-6]. Better understanding of the pathogenesis of LC and the development of molecular and cellular biomarkers for early detection is very important for improving LC therapeutic outcome [7-9], and 
development of a serum biomarker that can be applied simply and easily in clinical practice as a complementary tool for the prediction of prognostic outcome would be useful in the LC setting.

All cell types subjected to chemical, physical activation (thrombin, endotoxin, or shear stress) or apoptosis (growth factor deprivation or apoptotic inducers) can release plasma membrane fragments, called microparticles (MPs) [10]. MPs are small membrane-enclosed vesicles that are derived from the plasma membrane and released by activated and/or apoptotic cells. MPs are very small—ranging in size from $0.1 \mu \mathrm{m}$ to $1.0 \mu \mathrm{m}$ - and have been previously identified to circulate in the blood and have the ability to mediate inflammation and thrombosis [10-15]. MPs have differential effects on angiogenesis depending on their origin [10, 16-19]. MPs from platelets (PMPs) are proangiogenic; they promote capillary like structures and proangiogenic factor production $[10,13$, $16,18]$. In contrast, endothelial- and lymphocyte-derived MPs can be pro- or antiangiogenic depending on the stimuli used for their production [10].

Increased numbers of circulating MPs have been reported in a wide range of diseases [10], including acute coronary syndrome [20], infectious diseases [21], diabetes mellitus [22], uremia [23], hematologic malignancy [24, 25], and breast cancer [26]. Surprisingly, an association between circulating level of MPs and LC has seldom been reported [27]. Therefore the significance of MPs in LC remains uncertain. The aim of this study was to test (1) whether circulating levels of MPs are significantly higher in LC patients than in normal control subjects, (2) whether the circulating level of MPs is significantly higher in late stage LC patients than in early stage LC patients, (3) whether the circulating level of MPs differs among the patients with different disease statuses (i.e., disease controlled, disease progression and disease with no treatment (fresh case)), and (4) whether the circulating level of MPs is different among the different cell types of LC. This study further tested (5) whether this biomarker is predictive of intrapulmonary or distal metastasis of LC.

\section{Materials and Methods}

2.1. Patient Population, Data Collection, and Therapeutic Strategy. Image studies and pathological findings of all patients who presented with LC at the Kaohsiung Chang Gung Memorial Hospital were evaluated, and patients' eligibility for interventions, including surgery, adjunctive/palliative chemotherapy, irradiation therapy, and/or target therapy, was based on the 7th edition of the American Joint Committee on Cancer (AJCC) staging criteria [28]. LC was categorized into stages I, II, III, and IV according to the radiological findings based on the 7th edition of the AJCC staging criteria [28]. All the patients were enrolled in either the outpatient department or upon hospital admission for further evaluation and treatment. Additionally, early stage LC patients were enrolled only prior to surgical intervention.

Detailed in-hospital and follow-up data, including age, gender, chest X-ray findings, computed tomography, fibrobronchoscopic findings, bone scans or ultrasound studies, other image findings, and histological and pathological findings, were collected prospectively and entered into a computer database.

One hundred and thirty consecutive patients of all ages who were diagnosed with LC between April 2011 and February 2012 were prospectively enrolled into the study (stage I, $n=3$ (2.3\%); stage II, $n=3$ (2.3\%); stage IIIa, $n=17(27.7 \%)$, stage IIIb, $14.6 \%$ (19), $n=49$; stage IV, $n=88(67.7 \%)$ ) (Table 1).

Thirty age- and gender-matched healthy controls were also studied. Informed consent was obtained from each study subject and all participants have provided their written informed consent to participate in this study. The whole study protocol was approved by the Institutional Review Committee on Human Research at Kaohsiung Chang Gung Memorial Hospital (the IRB number: 100-0204C). This clinical investigation has been conducted according to the principles expressed in the Declaration of Helsinki.

To circumvent other potential influences on measurement of circulating level of MPs, patients with one or more of the following were excluded: recent surgery or trauma during the preceding 2 months, refusal to participate in the study, other coexistent malignances, severe organ disease other than LC, chronic kidney disease (CKD > stage III), liver cirrhosis, hematologic disorders, congestive heart failure, current use of antiplatelet agents, history of febrile disorders, acute or chronic inflammatory disease other than LC during the study period, or a history of autoimmune diseases with or without immunosuppressive therapy.

2.2. Categorized Circulating Microparticles. The circulating MPs were categorized into (1) platelet-derived activated MPs (PDAc-MPs) (CD31 $\left.{ }^{+} \mathrm{CD}_{2} 2 \mathrm{~b}^{+} \mathrm{AN}-\mathrm{V}^{-}\right)$, (2) plateletderived apoptotic MPs (PDAp-MPs) $\left(\mathrm{CD} 31^{+} \mathrm{CD}^{2} \mathrm{~b}^{+} \mathrm{AN}-\right.$ $\mathrm{V}^{+}$), (3) endothelial-derived activated MPs (EDAc-MPs) $\left(\mathrm{CD}^{+} 1^{+} \mathrm{CD}_{2} \mathrm{~b}^{-} \mathrm{AN}-\mathrm{V}^{-}\right)$, and (4) endothelial-derived apoptotic MPs (EDAp-MPs) (CD31 $\left.{ }^{+} \mathrm{CD} 42 \mathrm{~b}^{-} \mathrm{AN}-\mathrm{V}^{+}\right)$based on a previous report [29] with some modifications.

2.3. Blood Sampling for Biochemistry, Blood Cell Count Study, and Flow Cytometric Quantification of Plasma Levels of Microparticles. Blood samples were obtained once at 9:00 am from study subjects and once from 30 healthy control subjects who participated in a health screening program in the Health Clinic of Kaohsiung Chang Gung Memorial Hospital.

White blood cell (WBC) counts, biochemistry, and electrolyte levels were performed using standard laboratory methods.

Peripheral blood was collected in acid citrate dextrose (ACD) vacutainer tubes. The peripheral blood $(1.5 \mathrm{~mL})$ was centrifuged at $2500 \mathrm{~g}$ at $4^{\circ} \mathrm{C}$ for $15 \mathrm{~min}$ without acceleration or break to prepare platelet-rich plasma. The $250 \mu \mathrm{L}$ plasma samples were thawed and centrifuged for $10 \mathrm{~min}$ at $19,800 \mathrm{~g}$ at $4^{\circ} \mathrm{C}$ and then collected for investigation of microparticles (MPs) smaller than $1.0 \mu \mathrm{m}$.

Size calibration was conducted with $1.0 \mu \mathrm{m}$ beads (Invitrogen, Carlsbad, CA). The MP pellet was resuspended with $150 \mu \mathrm{L}$ of Annexin-V binding buffer (BD Biosciences). All buffers were sterile-filtered with a $0.2 \mu \mathrm{m}$ filter. The $100 \mu \mathrm{L}$ MPs were then incubated in a TruCOUNT tube (BD 
TABLE 1: Baseline characteristics of study patients and normal control subjects.

\begin{tabular}{|c|c|c|c|}
\hline Variable & Study patients $(n=130)$ & Normal control $(n=30)$ & $P$ \\
\hline Age (years) & $64.34 \pm 12.12$ & $64.01 \pm 8.56$ & 0.863 \\
\hline Male gender & $55.5 \%(71)$ & $50 \%(15)$ & 0.824 \\
\hline Body mass index $\left(\mathrm{kg} / \mathrm{m}^{2}\right)$ & $22.64 \pm 3.99$ & $23.56 \pm 3.09$ & 0.169 \\
\hline Stage I & $2.3 \%(3)$ & - & \\
\hline Stage II & $2.3 \%(3)$ & - & \\
\hline Stage IIIa & $27.7 \%(17)$ & - & \\
\hline Stage IIIb & $14.6 \%(19)$ & - & \\
\hline Stage IV & $67.7 \%(88)$ & - & \\
\hline Surgical intervention for LC & $0 \%(0)$ & - & \\
\hline History of smoking & $45.4 \%(59)$ & - & \\
\hline Hypertension & $18.5 \%(24)$ & - & \\
\hline Hypercholesterolemia & $16.4 \%(21)$ & - & \\
\hline Diabetes mellitus & $9.2 \%(12)$ & - & \\
\hline $\mathrm{RBC}$ count $\left(\times 10^{6} / \mathrm{dL}\right)$ & $4.29 \pm 1.28$ & $4.89 \pm 0.55$ & 0.001 \\
\hline WBC count $\left(\times 10^{3} / \mathrm{dL}\right)$ & $7.43 \pm 2.99$ & $5.88 \pm 1.28$ & 0.001 \\
\hline Platelet count $\left(\times 10^{4} / \mathrm{dL}\right)$ & $23.78 \pm 9.84$ & $22.45 \pm 4.07$ & 0.185 \\
\hline Creatinine $(\mathrm{mg} / \mathrm{dL})$ & $1.02 \pm 0.54$ & $0.88 \pm 0.19$ & 0.167 \\
\hline AST (IU/L) & $98.98 \pm 683.77$ & $27.44 \pm 11.07$ & $<0.001$ \\
\hline $\operatorname{ALT}(\mathrm{IU} / \mathrm{L})$ & $51.22 \pm 223.41$ & $30.56 \pm 16.83$ & $<0.001$ \\
\hline Ac sugar $(\mathrm{mg} / \mathrm{dL})$ & $131.51 \pm 62.9$ & $105.27 \pm 5.47$ & 0.001 \\
\hline $\mathrm{CD} 1^{+} \mathrm{CD} 42 \mathrm{~b}^{-} \mathrm{AN}-\mathrm{V}^{+}$(counts $\left./ \mathrm{mL}\right)^{\dagger}$ & $783(306-2014)$ & 457 (189-1127) & 0.012 \\
\hline $\mathrm{CD} 31^{+} \mathrm{CD} 42 \mathrm{~b}^{-} \mathrm{AN}-\mathrm{V}^{-}$(counts $\left./ \mathrm{mL}\right)^{\dagger}$ & $6969(2745-15206)$ & $5851(3619-10105)$ & 0.046 \\
\hline $\mathrm{CD} 31^{+} \mathrm{CD}_{2} 2 \mathrm{~b}^{+} \mathrm{AN}-\mathrm{V}^{+}(\text {counts } / \mathrm{mL})^{\dagger}$ & $15669(6020-50968)$ & 9817 (3867-38418) & 0.001 \\
\hline $\mathrm{CD} 31^{+} \mathrm{CD}_{2} 2 \mathrm{~b}^{+} \mathrm{AN}-\mathrm{V}^{-}$(counts $\left./ \mathrm{mL}\right)^{\dagger}$ & $25069(12224-51123)$ & $14923(8293-20839)$ & 0.001 \\
\hline
\end{tabular}

Data are expressed as mean \pm SD or $\%$ (number).

LC: lung cancer; BC: right blood cell count; WBC: white blood cell count; AST: aspartate aminotransferase; ALT: alanine aminotransferase.

${ }^{\dagger} \mathrm{CD} 31^{+} \mathrm{CD} 42 \mathrm{~b}^{-} \mathrm{AN}-\mathrm{V}^{+}$: endothelial-derived apoptotic microparticle (MP); $\mathrm{CD} 31^{+} \mathrm{CD} 42 \mathrm{~b}^{-} \mathrm{AN}_{-} \mathrm{V}^{-}$: endothelial-derived activated $\mathrm{MP}$; $\mathrm{CD} 31^{+} \mathrm{CD} 42 \mathrm{~b}^{+} \mathrm{AN}-$

$\mathrm{V}^{+}$: platelet-derived apoptotic MP; $\mathrm{CD} 31^{+} \mathrm{CD} 42 \mathrm{~b}^{+} \mathrm{AN}-\mathrm{V}^{-}$: platelet-derived activated MP.

Biosciences) with fluorescent monoclonal antibodies: (1) phycoerythrin- (PE-) labeled anti-CD31 (BD Biosciences), (2) fluorescein-isothiocyanate-labeled anti-Annexin-V (BD Biosciences), and (3) phycoerythrin-Cy5-(PE-Cy5-) labeled anti-CD42b (BD Biosciences). The samples were incubated in the dark for $15 \mathrm{~min}$ at room temperature. The samples were then analyzed on a FC500 flow cytometer (Beckman Coulter) after $400 \mu \mathrm{L}$ Annexin-V binding buffer was added. The absolute count of MPs was measured setting the stop condition for TruCount beads at 10,000 events.

2.4. Definitions. Assessment of the change in tumor burden was utilized to determine whether the tumor responded or not to the therapy. Accordingly, the chest computed tomography (CT) scans were performed at baseline and at three cycles (every 12-week interval) of therapy to determine the status of the disease. The criteria of tumor measurement were based on the current guideline of Response Evaluation Criteria in Solid Tumors (RECIST) [30], including complete response, partial response, stable disease, and progressive disease. Accordingly, in the current study, we categorized the disease statuses as (1) disease controlled, (2) disease progression, and (3) disease without treatment. "Disease controlled" status was defined as disease after treatment with regression and in a stable situation (i.e., including complete response, partial response, and stable disease). "Disease progression" was defined as disease unresponsive to therapy (complete course of treatment with tumor growing or disease metastasis). "Disease without treatment" was defined as a fresh case that was enrolled prior to treatment.

2.5. Statistical Analysis. Data were expressed as means \pm SD. Continuous variables were analyzed using independent $t$ tests and categorical variables were analyzed using the chisquare test. Statistical analysis was performed using SPSS statistical software for Windows version 13 (SPSS for Windows, version 13; SPSS Inc., IL). A $P$ value of $<0.05$ was considered statistically significant.

\section{Results}

3.1. Baseline Characteristics of Study Patients and Normal Controls. The baseline characteristics of both groups are shown in Table 1. Age, gender, and body mass index did not differ between study patients and normal controls. Additionally, the creatinine level and platelet count were similar between study patients and normal controls. However, the red blood cell count was insignificantly lower, whereas the white blood 
TABLE 2: Baseline variables and laboratory findings for early and late stage lung cancer patients.

\begin{tabular}{|c|c|c|c|}
\hline Variables & Early stage* $^{*}(n=23)$ & Late stage $^{*}(n=107)$ & $P$ \\
\hline Age (years) & $65.39 \pm 14.52$ & $64.11 \pm 11.67$ & 0.649 \\
\hline Male gender & $78.3 \%(18)$ & $49.5 \%(53)$ & 0.02 \\
\hline Smoking & $60.9 \%(14)$ & $42.1 \%(45)$ & 0.112 \\
\hline Hypertension & $21.7 \%(5)$ & $17.8 \%(19)$ & 0.767 \\
\hline Total cholesterol (mg/dL) & $197 \pm 71$ & $173 \pm 42$ & 0.469 \\
\hline Diabetes mellitus & $8.7 \%(2)$ & $9.3 \%(10)$ & 1.0 \\
\hline Body mass index $\left(\mathrm{kg} / \mathrm{m}^{2}\right)$ & $23.74 \pm 4.52$ & $22.39 \pm 3.84$ & 0.193 \\
\hline $\mathrm{RBC}$ count $\left(\times 10^{6} / \mathrm{dL}\right)$ & $4.37 \pm 0.53$ & $4.28 \pm 1.39$ & 0.770 \\
\hline WBC count $\left(\times 10^{3} / \mathrm{dL}\right)$ & $7.091 \pm 2.54$ & $7.504 \pm 3.08$ & 0.550 \\
\hline Platelet count $\left(\times 10^{4} / \mathrm{dL}\right)$ & $21.74 \pm 7.95$ & $24.22 \pm 10.18$ & 0.273 \\
\hline Creatinine $(\mathrm{mg} / \mathrm{dL})$ & $0.96 \pm 0.22$ & $1.03 \pm 0.59$ & 0.586 \\
\hline AST (IU/L) & $22(19-30)$ & $27(22-36)$ & 0.373 \\
\hline ALT (IU/L) & $18(13-27)$ & $21(16-36)$ & 0.169 \\
\hline Ac sugar (mg/dL) & $142.39 \pm 54.38$ & $129.18 \pm 64.65$ & 0.422 \\
\hline $\mathrm{CEA}(5 \mathrm{mg} / \mathrm{mL})$ & $11.96 \pm 22.24$ & $92.24 \pm 328.11$ & 0.243 \\
\hline $\mathrm{CD} 31^{+} \mathrm{CD} 42 \mathrm{~b}^{-} \mathrm{AN}-\mathrm{V}^{+}(\text {counts } / \mathrm{mL})^{\dagger}$ & $1086(297-3348)$ & $716(306-1872)$ & 0.158 \\
\hline $\mathrm{CD} 1^{+} \mathrm{CD} 42 \mathrm{~b}^{-} \mathrm{AN}-\mathrm{V}^{-}$(counts $\left./ \mathrm{mL}\right)^{\dagger}$ & 7935 (3434-23079) & $6546(2685-13634)$ & 0.169 \\
\hline $\mathrm{CD} 1^{+} \mathrm{CD} 42 \mathrm{~b}^{+} \mathrm{AN}-\mathrm{V}^{+}(\text {counts } / \mathrm{mL})^{\dagger}$ & $19162(5311-174196)$ & $15357(6221-45201)$ & 0.407 \\
\hline $\mathrm{CD} 31^{+} \mathrm{CD}_{2} 2 \mathrm{~b}^{+} \mathrm{AN}-\mathrm{V}^{-}(\text {counts } / \mathrm{mL})^{\dagger}$ & $46846(17141-66585)$ & $22776(12049-44329)$ & 0.031 \\
\hline
\end{tabular}

Data are expressed as mean \pm SD or \% (number).

LC: lung cancer; RBC: white blood cell count; WBC: white blood cell count; AST: aspartate aminotransferase; ALT: alanine aminotransferase; CEA: carcinoembryonic antigen.

*Early stage: stages I, II, and IIIa (i.e., operable condition) lung cancer; late stage: stages IIIb and IV (inoperable) lung cancer.

${ }^{\dagger} \mathrm{CD} 31^{+} \mathrm{CD} 42 \mathrm{~b}^{-} \mathrm{AN}-\mathrm{V}^{+}$: endothelial-derived apoptotic microparticle (MP); $\mathrm{CD} 31^{+} \mathrm{CD} 42 \mathrm{~b}^{-} \mathrm{AN}_{-} \mathrm{V}^{-}$: endothelial-derived activated $\mathrm{MP}$; $\mathrm{CD} 31^{+} \mathrm{CD} 42 \mathrm{~b}^{+} \mathrm{AN}-$ $\mathrm{V}^{+}$: platelet-derived apoptotic MP; $\mathrm{CD} 31^{+} \mathrm{CD} 42 \mathrm{~b}^{+} \mathrm{AN}-\mathrm{V}^{-}$: platelet-derived activated MP.

cell count was significantly higher in study patients than in normal controls. Additionally, the circulating levels of aspartate aminotransferase (AST) and alanine aminotransferase (ALT), two indices of liver function, were significantly elevated in study patients in comparison with normal subjects. Furthermore, the serum level of fasting blood sugar was significantly higher in study patients than in normal controls.

No incidence of smoking, hypertension, hypercholesterolemia, or diabetes mellitus or indices of coronary artery disease (CAD) risk factors were present in the normal controls. In the study patients, a history of smoking was most prevalent among CAD risk factors, followed by hypertension and hypercholesterolemia. Incidence of diabetes mellitus was the lowest CAD risk factor in the study patients.

The incidence of early stage (stage I to IIIa, that is, operable) LC was significantly lower than the late stage (stage $\geqq$ IIIb, that is, inoperable) LC among the study patients $(P<$ $0.001)$. An important finding was that the circulating levels of PDAc-MPs, PDAp-MPs, EDAc-MPs, and EDAp-MPs were substantially higher in LC patients than in normal controls. These findings might suggest that the LC patients had faster turnover of endothelial cells and shorter half life of platelets.

3.2. Comparison of Baseline Characteristics and Laboratory Findings between Early and Late Stage Lung Cancer Patients. There were no differences in terms of age, gender, CAD risk factors, body mass index, red and white blood cell counts, and platelet count between the early and late stage LC patients
(Table 2). Additionally, the serum levels of AST, ALT, creatinine, carcinoembryonic antigen (CEA), and fasting blood sugar were similar between the two groups. Furthermore, circulating levels of PDAp-MPs, EDAc-MPs, and EDAp-MPs did not differ between these two groups of patients. However, the circulating level of PDAc-MPs was lower in late stage LC patients than in early stage LC patients.

3.3. Comparison of Circulating Levels of Microparticles in Patients with Different Treatment Statuses. To determine whether the circulating levels of MPs were significantly different in LC patients with different disease statuses, plots of different kinds of MPs to different disease statuses were performed. The results (Figure 1) demonstrated that the circulating levels of PDAc-MPs, PDAp-MPs, EDAc-MPs, or EDAp-MPs did not significantly correlate with the presentation of any disease status (all $P>0.2$ ).

3.4. Comparison of Circulating Levels of Microparticles in Patients with Different Metastatic Statuses. To elucidate whether the circulating levels of the MPs were significantly associated with LC metastasis, plots of different kinds of MPs with no metastasis (M0), intrapulmonary metastasis (i.e., lung and pleural) (M1a), and extrapulmonary metastasis (i.e., distal metastasis) (M1b) were performed. The results (Figure 2) demonstrated that the circulating levels of PDAc-MPs, PDAp-MPs, EDAc-MPs, and EDAp-MPs did not 


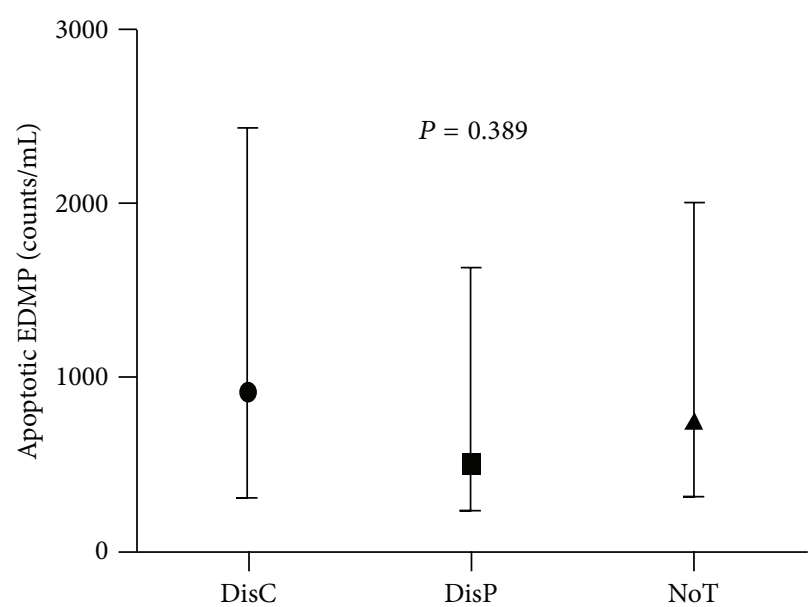

(a)

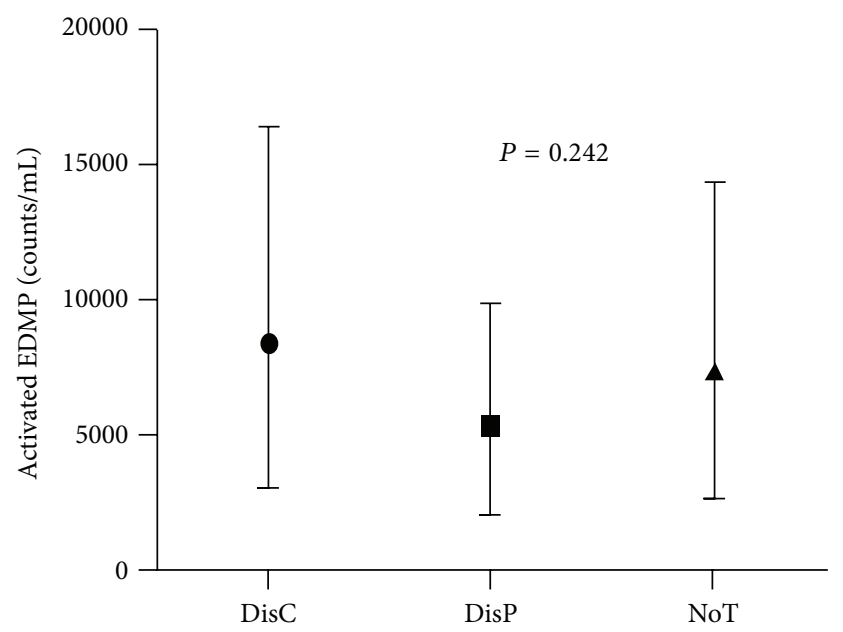

(c)

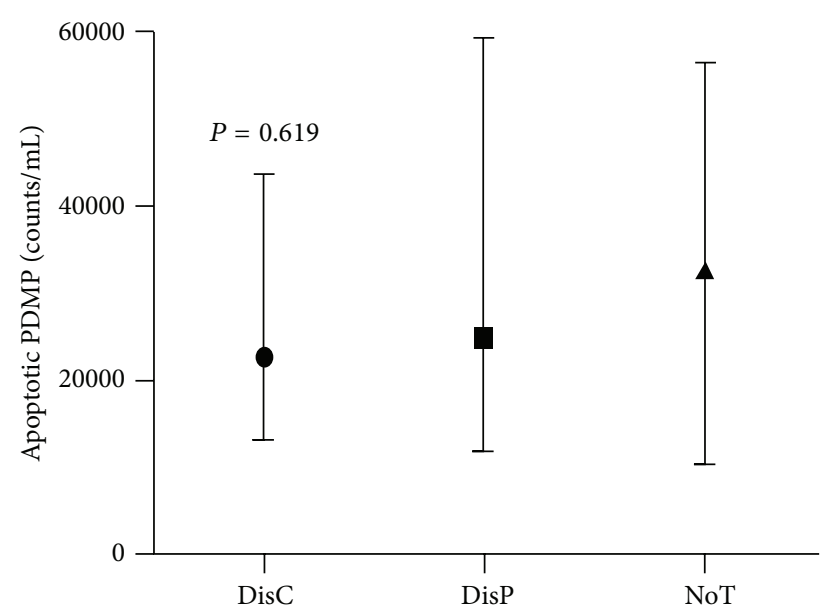

(b)

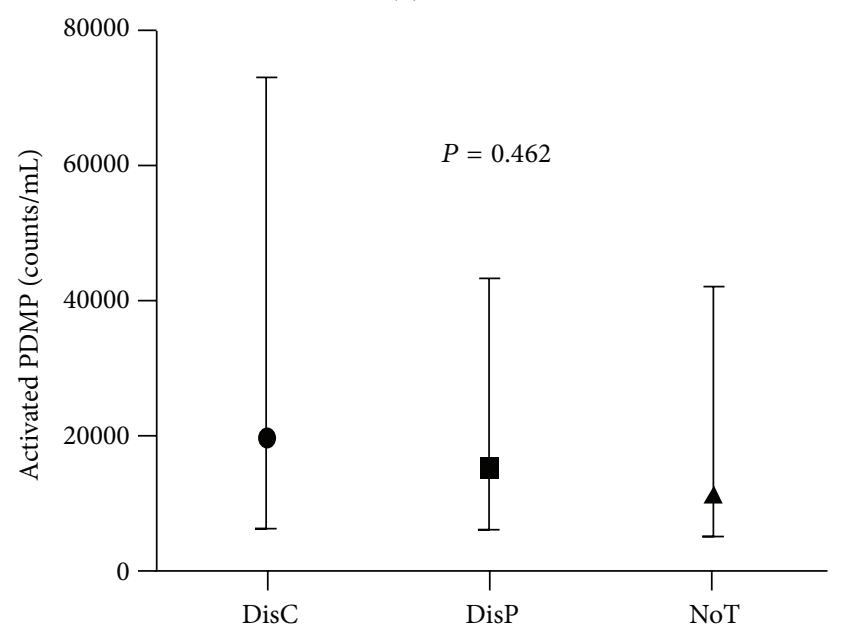

(d)

FIGURE 1: Comparison of plasma levels of microparticles (MPs) with different treatment statuses. (a) Apoptotic EDMP plotted against disease status, $P=0.389$; (b) apoptotic PDMP plotted against disease status, $P=0.619$; (c) activated EDMP plotted against disease status, $P=$ 0.242; (d) activated PDMP plotted against disease status, $P=0.462$. DisC $=$ disease control $(n=66)$; Dis $P=$ disease progression $(n=26)$; NoT $=$ disease with no treatment $(n=38)$. In these plots, the dot within the line represents the median values; the upper and lower lines represent the 25th and 75th percentiles, respectively. The analysis was performed using the Kruskal-Wallis test. EDMP $=$ endothelium-derived microparticles; PDMP = platelet-derived microparticles.

significantly correlate with any disease presentation status (i.e., with or without metastasis) (all $P>0.5$ ).

\subsection{Comparison of Circulating Levels of Microparticles in} Patients with Different Lung Cancer Cell Types. To estimate whether the circulating level of MPs was significantly correlated with different $\mathrm{LC}$ cell types according to the pathological findings, plots of different kinds of MPs against different kinds of LC (adenocarcinoma, squamous cell carcinoma, and small cell carcinoma) were constructed. The results (Figure 3) showed that the circulating levels of PDAc-MPs, PDApMPs, or EDAc-MPs did not significantly correlate with any different LC cell type (all $P>0.1$ ). However, the circulating level of EDAp-MPs was significantly associated with the different LC cell types $(P=0.045)$. Further analysis showed that this biomarker was significantly more associated with small cell carcinoma and significantly less associated with squamous cell carcinoma.
3.6. The Median Overall Survival of Stage IV Patients and the Incidence of Clinical Progression among 130 Patients. In the current study, the patients were consecutively followed up from enrollment time (i.e., from April 2011) up to December 2012. Thus, the median follow-up time was 15 months (range from 3 months to 37 months) in 88 patients with stage IV lung cancer. During this follow-up period, 46 patients were dead and another 42 patients were still alive. Additionally, within this enrollment and follow-up period, among the 130 study patients, we found that 26 patients had clinical progression, 67 patients were in the status of disease control, and 27 patients were the fresh cases.

\section{Discussion}

This study investigated whether circulating level of MPs could be potentially useful as a biomarker in daily clinical practice to predict severity and prognostic outcome in LC patients. 


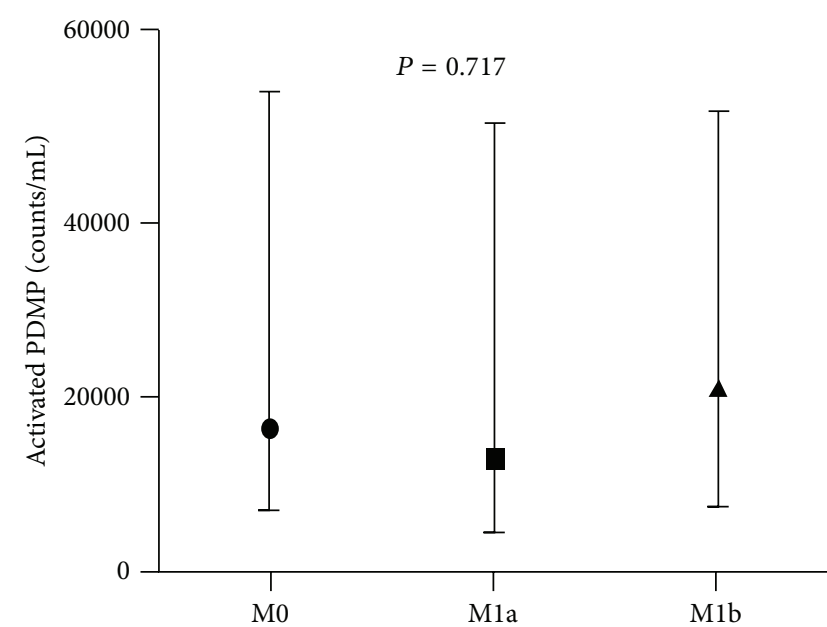

(a)

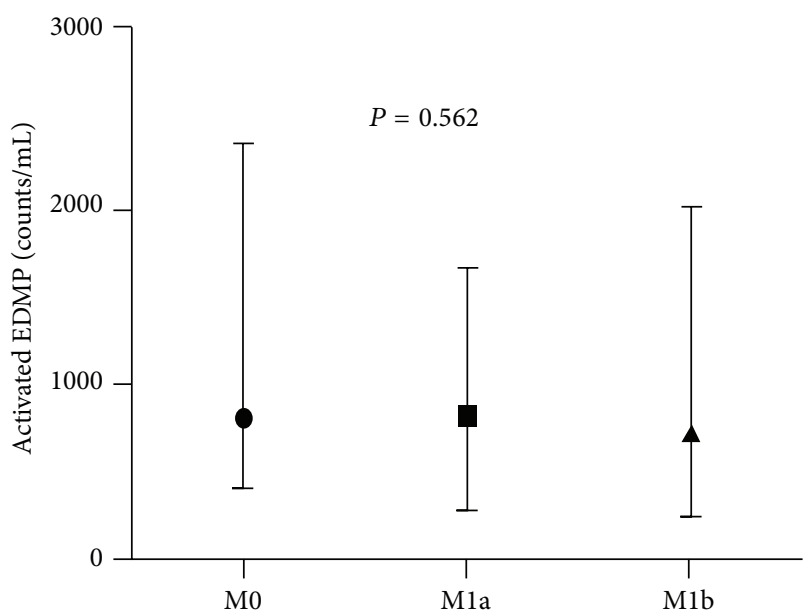

(c)

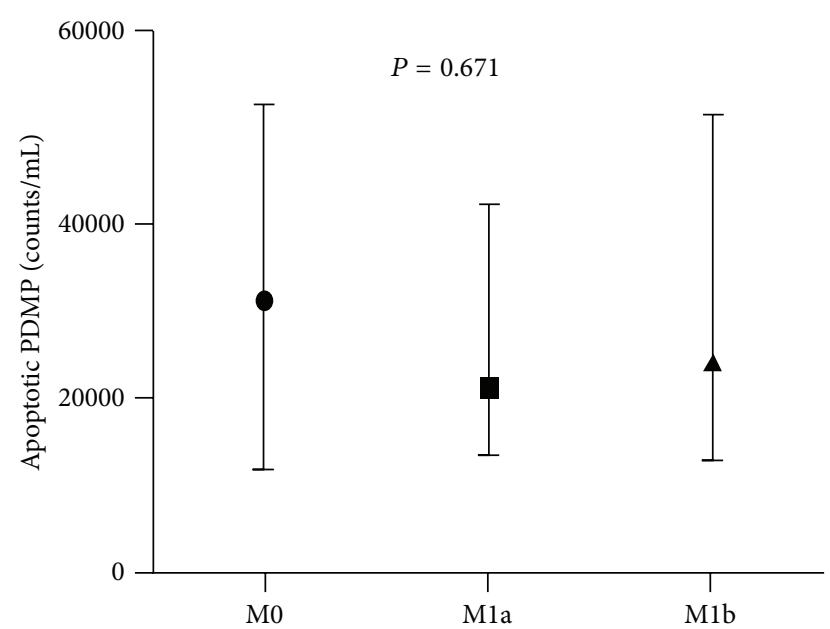

(b)

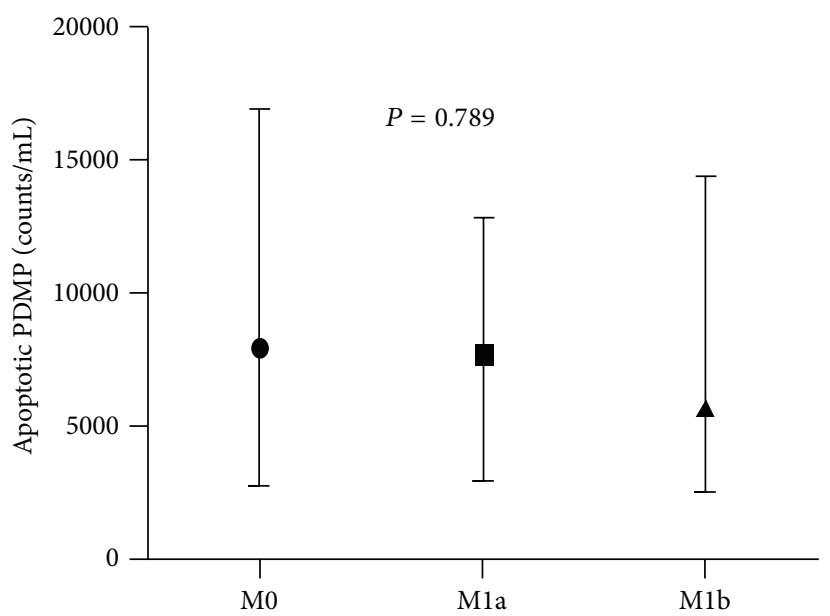

(d)

FIGURE 2: Comparison of plasma levels of microparticles to the presence or absence of lung cancer metastasis. (a) Apoptotic EDMP plotted against presence or absence of lung cancer (LC) metastasis, $P=0.717$; (b) apoptotic PDMP plotted against presence or absence of LC metastasis, $P=0.671$; (c) activated EDMP plotted against presence or absence of LC metastasis, $P=0.562$; (d) activated PDMP plotted against presence or absence of LC metastasis, $P=0.462$. M0 = no metastasis $(n=40)$; Mla $=$ intrathoracic metastasis (i.e., lung and pleural; $n=31) ; \mathrm{Mlb}=$ extrathoracic metastasis $(n=59)$. In these plots, the dot within the line represents the median values; the upper and lower lines represent the 25 th and 75 th percentiles, respectively. The analysis was performed using the Kruskal-Wallis test. EDMP = endothelium-derived microparticles; PDMP = platelet-derived microparticles.

The findings have several clinical implications. First, the circulating levels of MPs, including PDAc-MPs, PDAp-MPs, EDAc-MPs, and EDAp-MPs, were significantly higher in LC patients than in normal control subjects. Second, there was no significant correlation between circulating levels of these biomarkers and the stages of the LC. Third, circulating levels of MPs were likewise not significantly associated with the presence or absence of metastasis. Finally, only the circulating level of EDAp-MPs was found to be significantly correlated with different LC cell types.

\subsection{Level of Circulating Endothelial Cell-Derived and Platelet-} Derived MPs in Lung Cancer Patients. One important finding in the present study is that the circulating levels of MPs which were derived from both endothelial cells and platelets were found to be remarkably higher in LC patients than in normal controls. MPs are detectable in plasma from healthy subjects and, in general, their circulating levels are enhanced in pathological situations. Previous reports have shown that an increase in circulating MPs frequently occurs in a wide range of diseases [10] such as acute coronary syndrome [20], infectious diseases [21], diabetes mellitus [22], uremia [23], hematologic malignancy $[24,25]$ and breast cancer [26]. Therefore, our finding is consistent with the findings of previous studies $[10,16,20-23]$. Of importance, the current findings and findings from previous studies [10, 16, 20-23] suggest that an increase in circulating level of MPs may be associated with the activity of disease entity.

It is well recognized that increased angiogenesis/vasculogenesis and neovascularization for nutrition and blood supply always occur in solid cancers, including lung cancer. MPs can affect angiogenesis by inducing changes in 


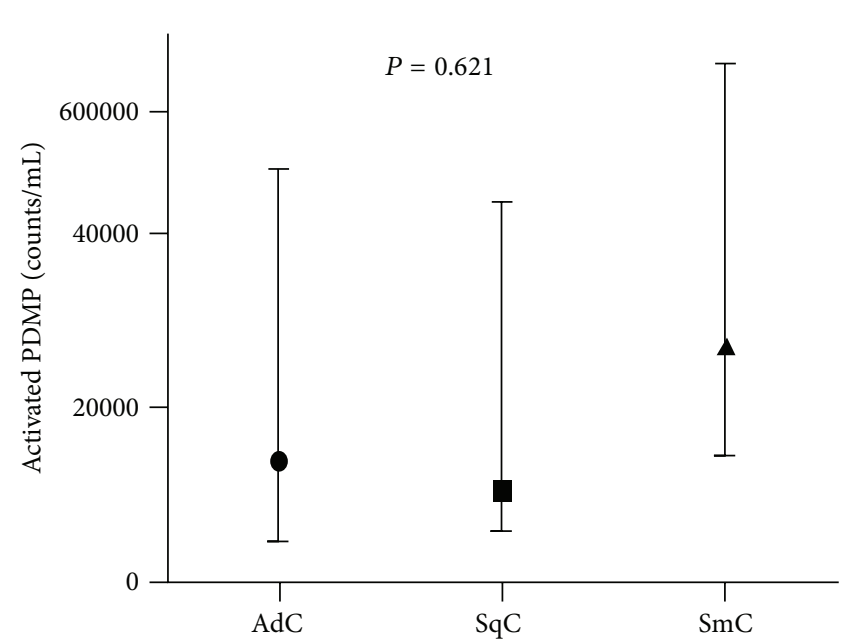

(a)

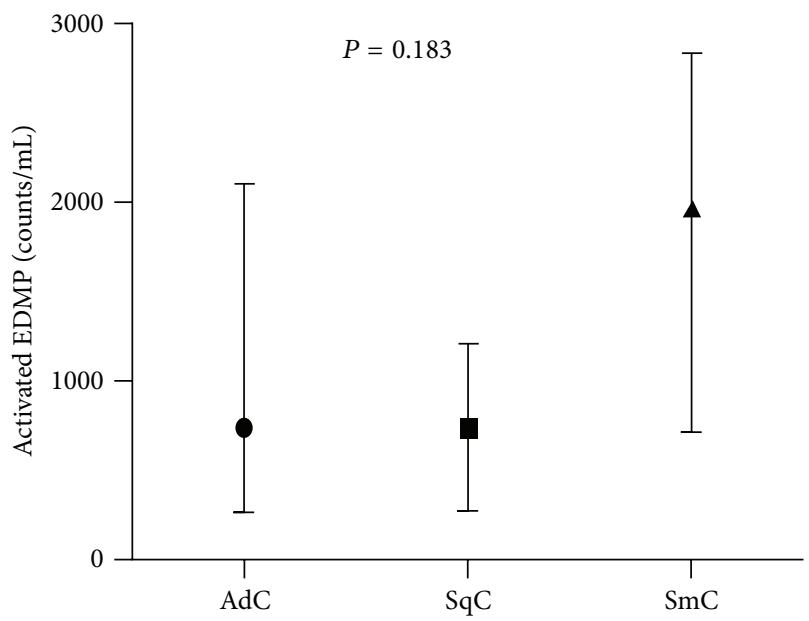

(c)

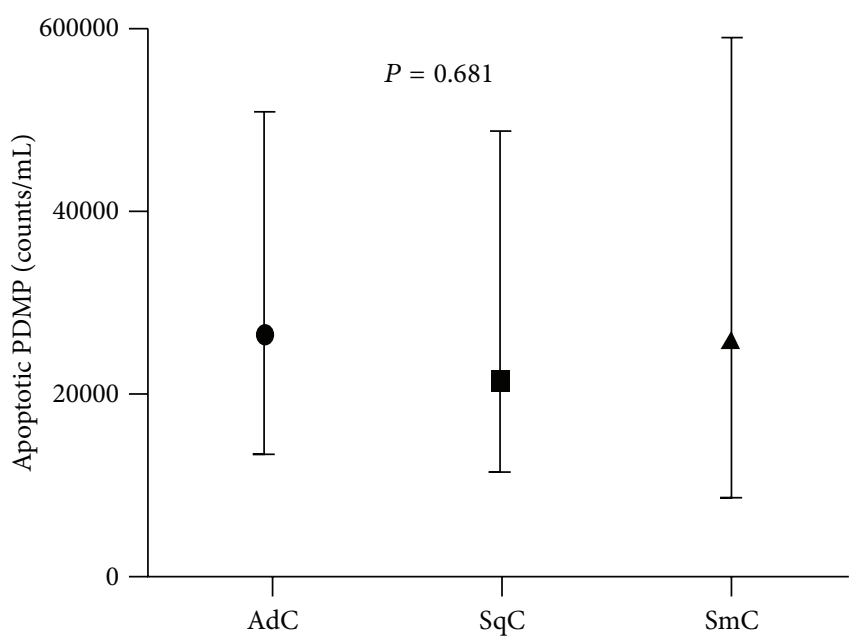

(b)

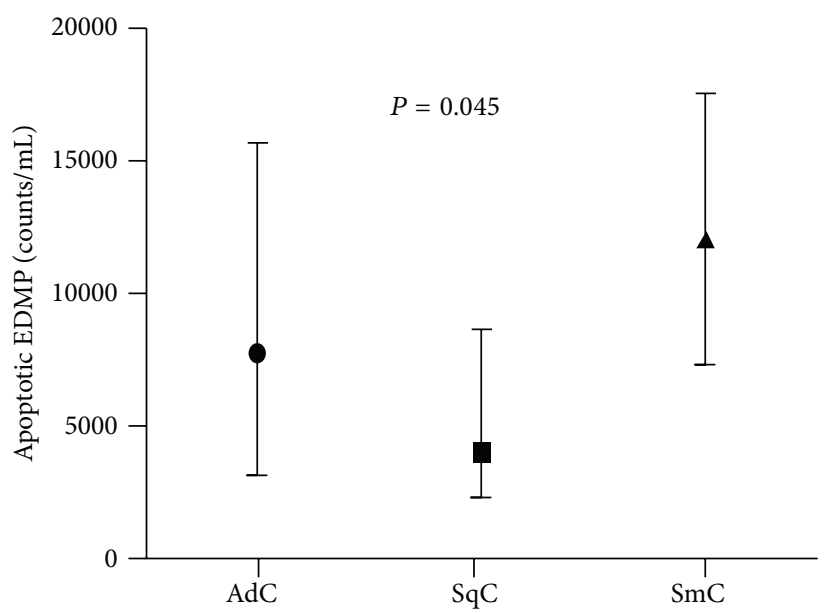

(d)

FIGURE 3: Illustration of the comparison of plasma levels of microparticles to different cell types of lung cancer (LC). (a) Apoptotic EDMP against different cell types of LC, $P=0.621$; (b) apoptotic PDMP plotted against different cell types of LC, $P=0.681$; (c) activated EDMP plotted against different cell types of LC, $P=0.183$; (d) activated PDMP plotted against different cell types of LC, $P=0.045$. AdC $=$ adenocarcinoma $(n=91)$; SqC = squamous carcinoma $(n=32)$; SmC = small cell lung cancer $(n=7)$. In these plots, the dot within the line represents the median values; the upper and lower lines represent the 25 th and 75 th percentiles, respectively. The analysis was performed using the Kruskal-Wallis test. EDMP = endothelium-derived microparticles; PDMP = platelet-derived microparticles.

the secretome of endothelial cells, either increasing the production of proangiogenic factors or decreasing the production of antiangiogenic factors [31-33]. These findings [31-33] implicate that MPs are able to modify steps involved in angiogenesis, such as proliferation, migration, and adhesion of endothelial cells. These could explain why the circulating level of MPs was found to be increased in the lung cancer patients in the present study. Besides, we propose that the ability of lung cancer cells generating MPs suggests that they could play a critical role in angiogenesis facilitating metastasis, which is the main reason for explaining the levels of circulating microparticles increasing in lung cancer patients in our study.

Interestingly, MPs derived from platelets have been shown to be pro-angiogenic through the promotion of capillary-like structures and pro-angiogenic factor production
[10]. In the current study, levels of both PDAc-MPs and PDAp-MPs were found to be substantially higher in study patients than in normal subjects, consistent with previous studies [10].

\subsection{Predictive Value and Clinical Relevant Implication of Circulating Endothelial Cell-Derived and Platelet-Derived MPs in Lung Cancer. Although the levels of circulating MPs have frequently been reported to be increased in a variety of diseases [10, 16, 20-23, 26, 27], whether or not MPs could be useful in predicting disease outcomes in daily clinical practice is still not clear, especially in the LC setting. In the present study, importantly, no types of circulating MPs were found to be predictive of disease status, or metastatic status. Another negative but similarly important finding was that PDAc-MPs,}


PDAp-MPs, and EDAc-MPs were not significantly predictive of LC cell type.

To the best of our knowledge, only one previous study has mentioned the level and value of MPs in LC patients [27]. This study demonstrated that the circulating levels of both monocyte-derived MPs (MDMPs) and platelet-derived (PD) MPs were significantly higher in LC patients than in normal control subjects. Our current results support this finding [27]. However, the previous study reported that circulating levels of MDMPs and PDMPs were significantly higher in non-small-cell LC patients than in small cell LC patients. Conversely, our study revealed that only circulating level of EDAp-MPs was significantly associated with small cell rather than non-small-cell LC. Our results are, therefore, inconsistent with the finding of the previous study in this respect [27]. We are uncertain as to why there is a discrepancy between our study and the previous study [27]. Conceivably, differences between patient sample size and enrollment timing, the flow cytometry methodology for analysis of the MPs, the LC stage of the patients, the age distribution of the patients, and/or the treatment course of LC patients in our study and the previous study [27] may have affected the outcome.

In the present study, the circulating level of EDApMPs is lower in lung squamous cell carcinoma than in other cell types. As in a previous study report, EDAp-MPs may activate matrix metalloproteases, which are involved in the extracellular matrix degradation and the release of growth factors that play a crucial role in tissue remodeling, angiogenesis, and cancer spreading [34]. Lung squamous cell carcinoma most often arises centrally in larger bronchi, and while it often metastasizes to locoregional lymph nodes (particularly the hilar nodes) early in its course, it generally disseminates outside the thorax somewhat later than other cell types of lung cancer. Lung squamous cell carcinoma also tends to be slow growing; however, lung small cell carcinoma and lung adenocarcinoma may grow quickly and spread early in the course of the disease [35]. Therefore, lung squamous cell carcinoma is believed to be slower cancer spreading and lower extent of angiogenesis in host microenvironment than other cell types of lung cancer. It can be expected that circulating level of EDAp-MPs is lower in lung squamous cell carcinoma than in other cell types.

Interestingly, the circulating levels of EDAc-MPs, EDApMPs, and PDAp-MPs were relatively higher in early stage LC patients than in late stage LC patients although the results were not statistically significant. Of importance, the circulating level of PDAc-MPs was significantly higher in early stage LC patients than in late stage LC patients. These findings perhaps suggest that turnover of MPs is faster in early stage than in late stage LC patients. Of particular importance was that although our data showed that circulating levels of all types of MPs were significantly increased in LC patients, only circulating levels of EDAp-MPs were ascertained significantly correlated with small cell rather than non-small-cell LC types. This finding may be the most importantly clinical relevant implication in the current study and encourages the use of this kind of MPs as a predictive biomarker for in LC patients.

\section{Study Limitations}

This study was limited by the following factors. First, the sample size of this study was relatively small; thus, the conclusions based on the findings of the present study are tentative and care should be exercised in extrapolating the results of this study to the clinical setting. Second, the statuses of patients who were enrolled into the study were not identical at clinical presentation; that is, not all the patients had not received treatment at presentation. Therefore, the impact of circulating MPs on the prognostic outcome of LC patients could not be investigated in the present study.

\section{Conclusion}

This study demonstrated that circulating levels of MPs are significantly increased in LC patients, and circulating levels of EDAp-MPs were found significantly associated with different LC cell types. These findings suggest that a prospective clinical study with a larger sample size to investigate the possible usefulness of circulating MPs in predicting prognostic outcome in LC patients is warranted.

\section{Abbreviations}

$\begin{array}{ll}\text { ACD: } & \text { Acid citrate dextrose } \\ \text { AJCC: } & \text { American Joint of Committee on Cancer } \\ \text { ALT: } & \text { Alanine aminotransferase } \\ \text { AST: } & \text { Aspartate aminotransferase } \\ \text { CAD: } & \text { Coronary artery disease } \\ \text { CEA: } & \text { Carcinoembryonic antigen } \\ \text { CKD: } & \text { Chronic kidney disease } \\ \text { EDAc-MPs: } & \text { Endothelial-derived activated MPs } \\ \text { EDAp-MPs: } & \text { Endothelial-derived apoptotic microparticles } \\ \text { EDMPs: } & \text { Endothelial-derived microparticles; } \\ \text { LC: } & \text { Lung cancer } \\ \text { MDMPs: } & \text { Monocyte-derived microparticles } \\ \text { MPs: } & \text { Microparticles } \\ \text { PDAc-MPs: } & \text { Platelet-derived activated MPs } \\ \text { PDAp-MPs: } & \text { Platelet-derived apoptotic MPs } \\ \text { PDMPs: } & \text { Platelet-derived microparticles. }\end{array}$

\section{Conflict of Interests}

All of the authors declare no potential conflict of interests.

\section{Authors' Contribution}

Chia-Cheng Tseng and Chin-Chou Wang designed and performed the research. Li-Teh Chang, Steve Leu, and ChiaHung Yen technically supported the experiments of the flow cytometry. Tzu-Hsien Tsai, Huang-Chih Chang, Kuo-Tung Huang, and Chih-Hung Chen analyzed data. Chin-Chou Wang, Shih-Feng Liu, and Chia-Cheng Tseng wrote the paper. Cheng-Ta Yang, Hon-Kan Yip, and Meng-Chih Lin edited and reviewed the paper. All authors read and approved the final paper. Chia-Cheng Tseng and Chin-Chou Wang equally contributed to this study compared with the first 
author. Hon-Kan Yip and Meng-Chih Lin equally contributed to this study compared with the correspondence author.

\section{Acknowledgment}

This work was supported by a grant from the Chang Gung Memorial Hospital (CMRPG8B0011) to Chia-Cheng Tseng and (CMRPG880071) to Chin-Chou Wang.

\section{References}

[1] P. Pisani, D. M. Parkin, F. Bray, and J. Ferlay, "Estimates of the worldwide mortality from 25 cancers in 1990," International Journal of Cancer, vol. 83, pp. 870-873, 1999, Erratum: International Journal of Cancer, vol. 83, pp. 18-29, 1990.

[2] P. M. Marcus, E. J. Bergstralh, R. M. Fagerstrom et al., "Lung cancer mortality in the Mayo Lung Project: impact of extended follow-up," Journal of the National Cancer Institute, vol. 92, no. 16, pp. 1308-1316, 2000.

[3] R. Arriagada, B. Bergman, A. Dunant, T. Le Chevalier, J.-P. Pignon, and J. Vansteenkiste, "Cisplatin-based adjuvant chemotherapy in patients with completely resected non-small-cell lung cancer," The New England Journal of Medicine, vol. 350, no. 4, pp. 351-360, 2004.

[4] S. C. Spiro and G. A. Silvestri, "One hundred years of lung cancer," American Journal of Respiratory and Critical Care Medicine, vol. 172, no. 5, pp. 523-529, 2005.

[5] T. E. Stinchcombe and M. A. Socinski, "Maintenance therapy in advanced non-small cell lung cancer: current status and future implications," Journal of Thoracic Oncology, vol. 6, no. 1, pp. 174182, 2011.

[6] N. K. Veeramachaneni, R. H. Feins, B. J. Stephenson, L. J. Edwards, and F. G. Fernandez, "Management of stage IIIA non-small cell lung cancer by thoracic surgeons in North America," The Annals of Thoracic Surgery, vol. 94, pp. 922-928, 2012.

[7] R. S. Herbst, A. Onn, and A. Sandler, "Angiogenesis and lung cancer: prognostic and therapeutic implications," Journal of Clinical Oncology, vol. 23, no. 14, pp. 3243-3256, 2005.

[8] T. D. Chanin, D. T. Merrick, W. A. Franklin, and F. R. Hirsch, "Recent developments in biomarkers for the early detection of lung cancer: perspectives based on publications 2003 to present," Current Opinion in Pulmonary Medicine, vol. 10, no. 4, pp. 242-247, 2004.

[9] M. Hassanein, J. C. Callison, C. Callaway-Lane, M. C. Aldrich, E. L. Grogan, and P. P. Massion, "The state of molecular biomarkers for the early detection of lung cancer," Cancer Prevention Research, vol. 5, pp. 992-1006, 2012.

[10] H. A. Mostefai, R. Andriantsitohaina, and M. C. Martínez, "Plasma membrane microparticles in angiogenesis: role in ischemic diseases and in cancer," Physiological Research, vol. 57, no. 3, pp. 311-320, 2008.

[11] Z. Mallat, H. Benamer, B. Hugel et al., "Elevated levels of shed membrane microparticles with procoagulant potential in the peripheral circulating blood of patients with acute coronary syndromes," Circulation, vol. 101, no. 8, pp. 841-843, 2000.

[12] S. Martin, A. Tesse, B. Hugel et al., "Shed membrane particles from $\mathrm{T}$ lymphocytes impair endothelial function and regulate endothelial protein expression," Circulation, vol. 109, no. 13, pp. 1653-1659, 2004.

[13] M. C. Martínez, A. Tesse, F. Zobairi, and R. Andriantsitohaina, "Shed membrane microparticles from circulating and vascular cells in regulating vascular function," American Journal of Physiology-Heart and Circulatory Physiology, vol. 288, no. 3, pp. H1004-H1009, 2005.

[14] A. Mezentsev, R. M. H. Merks, E. O’Riordan et al., "Endothelial microparticles affect angiogenesis in vitro: role of oxidative stress," American Journal of Physiology-Heart and Circulatory Physiology, vol. 289, no. 3, pp. H1106-H1114, 2005.

[15] M. C. Martínez, F. Larbret, F. Zobairi et al., "Transfer of differentiation signal by membrane microvesicles harboring hedgehog morphogens," Blood, vol. 108, no. 9, pp. 3012-3020, 2006.

[16] S. Nomura, Y. Ozaki, and Y. Ikeda, "Function and role of microparticles in various clinical settings," Thrombosis Research, vol. 123, no. 1, pp. 8-23, 2008.

[17] R. F. A. Zwaal and A. J. Schroit, "Pathophysiologic implications of membrane phospholipid asymmetry in blood cells," Blood, vol. 89, no. 4, pp. 1121-1132, 1997.

[18] L. L. Horstman and Y. S. Ahn, "Platelet microparticles: a wideangle perspective," Critical Reviews in Oncology/Hematology, vol. 30, no. 2, pp. 111-142, 1999.

[19] S. Nomura, "Function and clinical significance of plateletderived microparticles," International Journal of Hematology, vol. 74, no. 4, pp. 397-404, 2001.

[20] S. Nomura, S. Uehata, S. Saito, K. Osumi, Y. Ozeki, and Y. Kimura, "Enzyme immunoassay detection of platelet-derived microparticles and RANTES in acute coronary syndrome," Thrombosis and Haemostasis, vol. 89, no. 3, pp. 506-512, 2003.

[21] K. Joop, R. J. Berckmans, R. Nieuwland et al., "Microparticles from patients with multiple organ dysfunction syndrome and sepsis support coagulation through multiple mechanisms," Thrombosis and Haemostasis, vol. 85, no. 5, pp. 810-820, 2001.

[22] N. Ogata, M. Imaizumi, S. Nomura et al., "Increased levels of platelet-derived microparticles in patients with diabetic retinopathy," Diabetes Research and Clinical Practice, vol. 68, no. 3, pp. 193-201, 2005.

[23] L. Daniel, F. Fakhouri, D. Joly et al., "Increase of circulating neutrophil and platelet microparticles during acute vasculitis and hemodialysis," Kidney International, vol. 69, no. 8, pp. 14161423, 2006.

[24] M. E. T. Tesselaar, F. P. H. T. M. Romijn, I. K. Van Der Linden, F. A. Prins, R. M. Bertina, and S. Osanto, "Microparticle-associated tissue factor activity: a link between cancer and thrombosis?" Journal of Thrombosis and Haemostasis, vol. 5, no. 3, pp. 520-527, 2007.

[25] S. Nomura, H. Kagawa, Y. Ozaki, M. Nagahama, C. Yoshimura, and S. Fukuhara, "Relationship between platelet activation and cytokines in systemic inflammatory response syndrome patients with hematological malignancies," Thrombosis Research, vol. 95, no. 5, pp. 205-213, 1999.

[26] A. Janowska-Wieczorek, L. A. Marquez-Curtis, M. Wysoczynski, and M. Z. Ratajczak, "Enhancing effect of platelet-derived microvesicles on the invasive potential of breast cancer cells," Transfusion, vol. 46, no. 7, pp. 1199-1209, 2006.

[27] S. Kanazawa, S. Nomura, M. Kuwana, M. Muramatsu, K. Yamaguchi, and S. Fukuhara, "Monocyte-derived microparticles may be a sign of vascular complication in patients with lung cancer," Lung Cancer, vol. 39, no. 2, pp. 145-149, 2003.

[28] S. B. Edge and C. C. Compton, "The American Joint Committee on Cancer: the 7th edition of the AJCC cancer staging manual and the future of TNM," Annals of Surgical Oncology, vol. 17, no. 6, pp. 1471-1474, 2010. 
[29] E. Dey-Hazra, B. Hertel, T. Kirsch et al., "Detection of circulating microparticles by flow cytometry: influence of centrifugation, filtration of buffer, and freezing," Vascular Health and Risk Management, vol. 6, no. 1, pp. 1125-1133, 2010.

[30] P. Therasse, S. G. Arbuck, E. A. Eisenhauer et al., "New guidelines to evaluate the response to treatment in solid tumors," Journal of the National Cancer Institute, vol. 92, no. 3, pp. 205216, 2000.

[31] R. Soleti, T. Benameur, C. Porro, M. A. Panaro, R. Andriantsitohaina, and M. C. Martínez, "Microparticles harboring Sonic Hedgehog promote angiogenesis through the upregulation of adhesion proteins and proangiogenic factors," Carcinogenesis, vol. 30, no. 4, pp. 580-588, 2009.

[32] A. Zernecke, K. Bidzhekov, H. Noels et al., "Delivery of microRNA-126 by apoptotic bodies induces CXCL12-dependent vascular protection," Science Signaling, vol. 2, no. 100, p. ra81, 2009.

[33] M. C. Martinez and R. Andriantsitohaina, "Microparticles in angiogenesis: therapeutic potential," Circulation Research, vol. 109, no. 1, pp. 110-119, 2011.

[34] F. Dignat-George and C. M. Boulanger, "The many faces of endothelial microparticles," Arteriosclerosis, Thrombosis, and Vascular Biology, vol. 31, no. 1, pp. 27-33, 2011.

[35] L. G. Collins, C. Haines, R. Perkel, and R. E. Enck, "Lung cancer: diagnosis and management," American Family Physician, vol. 75, no. 1, pp. 56-63, 2007. 


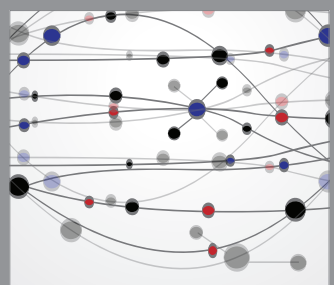

The Scientific World Journal
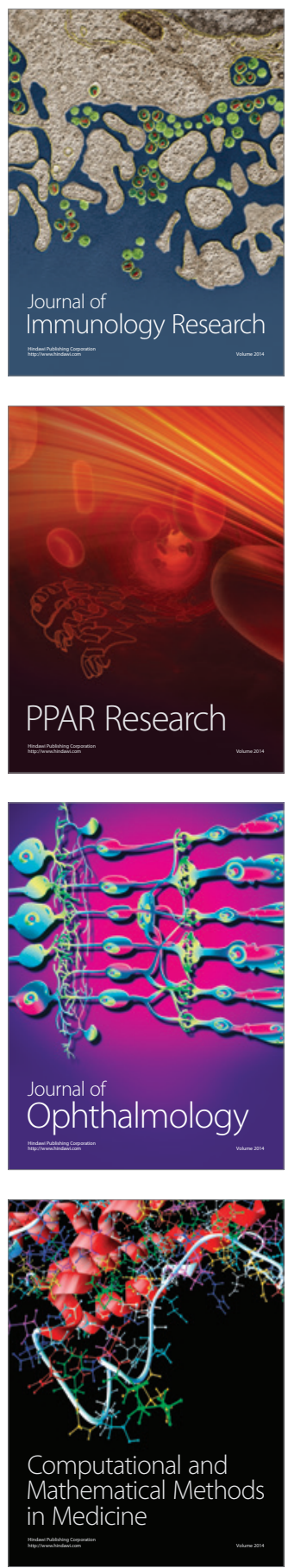

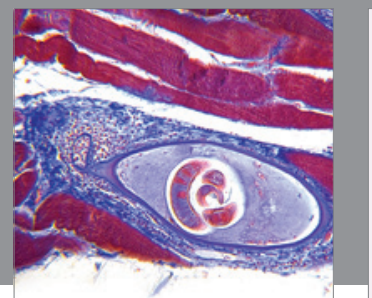

Gastroenterology

Research and Practice
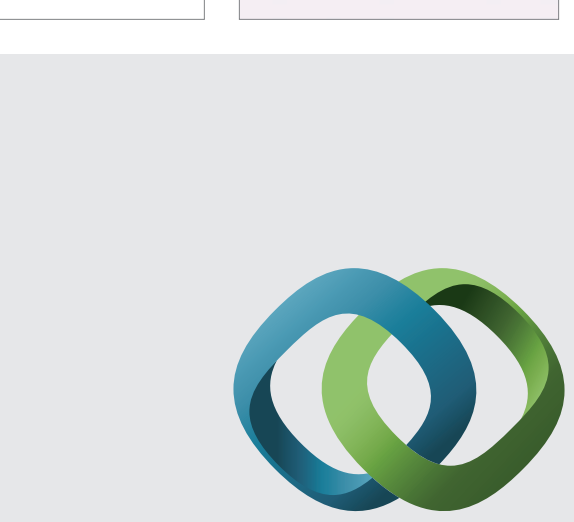

\section{Hindawi}

Submit your manuscripts at

http://www.hindawi.com
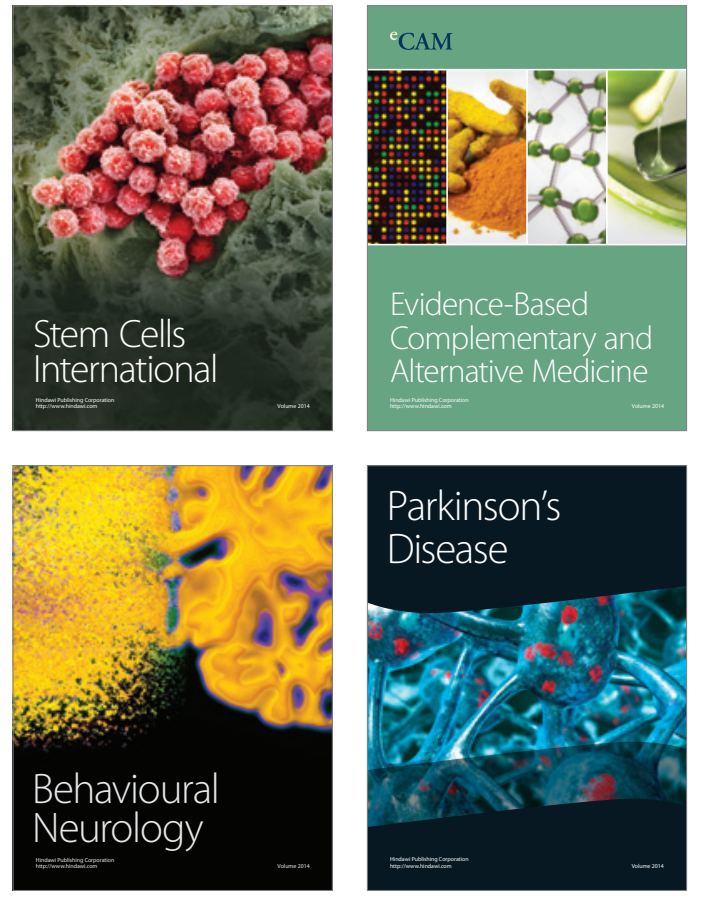
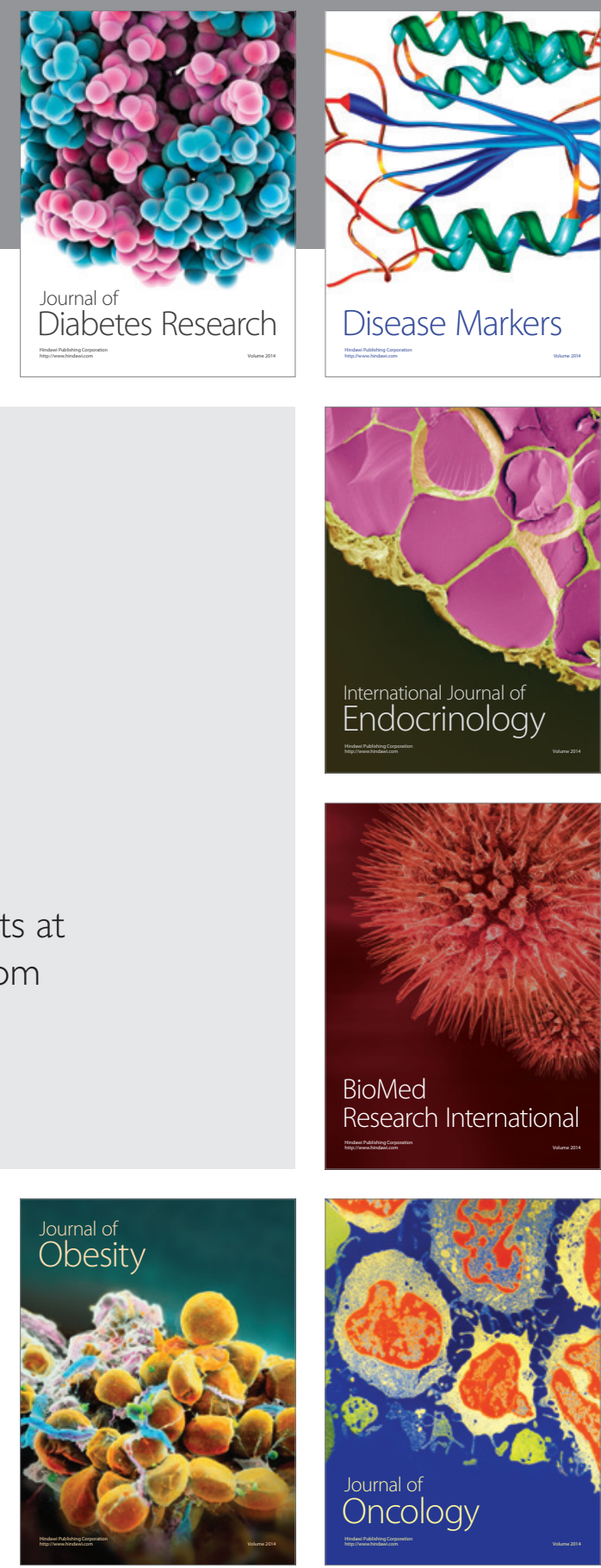

Disease Markers
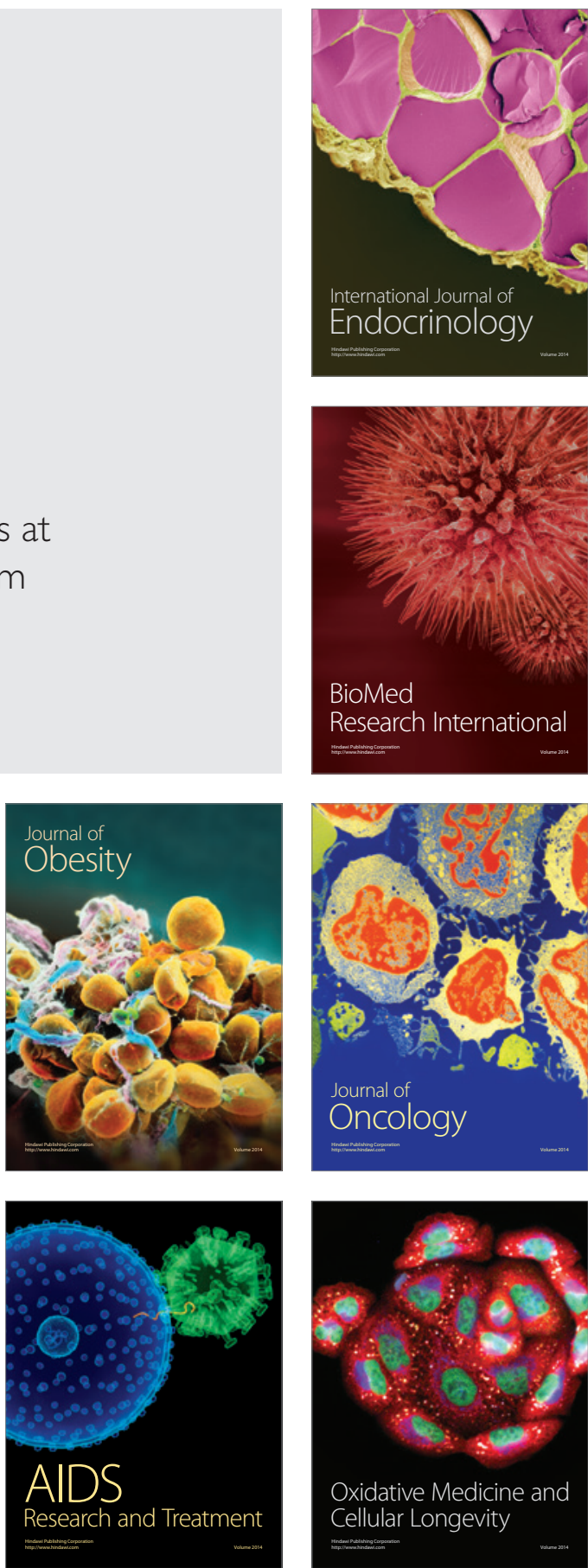